\title{
Spinning ion trajectories
}

In 1932, Ernest Lawrence invented the cyclotron-a particle accelerator using a static magnetic field in which charged particles follow an outward spiral, accelerated by a rapidly varying radiofrequency $(\mathrm{RF})$ field; for this work, he was awarded the Nobel Prize in Physics in 1939. At around that time, John Hipple was working for Westinghouse Electric Co. on the design of $90^{\circ}$ magnetic sector mass spectrometers (see Milestone 1). A few years later, after joining the US Bureau of Standards, Hipple combined his knowledge of magnetic sector mass spectrometers with the principles of cyclotron acceleration in a new device he called the omegatron.

In the first prototype, Hipple and two colleagues trapped hydrogen ions using a static electric potential and a magnetic field. Tuning the frequency of an additional RF field to resonance with the cyclotron frequency ensured that only ions with a desired charge-to-mass ratio would be accelerated. The ions would then be pushed along the exact outward-spiraling trajectory necessary for them to hit the detector. Today, this technique is known as ion cyclotron resonance (ICR) mass analysis and powers the highestperformance mass spectrometers.

By 1951, Hipple's team was already envisioning their omegatron as a powerful mass analyzer and discussing ways to improve its resolution using higher magnetic fields, better trapping and enhanced detection. Although trapping was improved in the subsequent development of magnetic ion traps, substantial improvements in detection had to wait until the 1974 work of Melvin Comisarow and Alan Marshall. Instead of detecting the charged particles directly,
Comisarow and Marshall measured the image current generated by the charges in the detector plates. Specifically, turning off the RF excitation causes bunches of ions to rotate at the cyclotron frequency. As the ions repeatedly pass the detector plates, they produce a free induction current that can be detected and subsequently converted to a frequency spectrum using the Fourier transform-hence the name 'Fourier transform ICR' (FT-ICR) given to the new technique.

With trapping and detection taken care of, the most obvious route to even higher resolution and mass accuracy was to increase the magnetic field. Thanks to superconducting magnets, these fields can go ever higher: the National High Magnetic Field Laboratory's FT-ICR mass spectrometers hold the current world record of 21 tesla, an impressive but very expensive achievement.

But were magnetic fields actually needed? Back in 1923, Kenneth Kingdon had described the trapping of charged particles in a simple electrostatic device - the Kingdon trapconsisting of a cylinder with a wire along its axis, with a voltage difference between the two. As Kingdon saw it, an ion would be imprisoned in the tube, forced to orbit to and fro around the axis until it lost its transverse velocity and collapsed into the wire.

In 2000, Alexander Makarov revisited this concept to create an equally simple and elegant design known as the orbitrap. Makarov replaced the wire with a spindle-shaped electrode and the cylinder with a barrel-like electrode. The ions would follow intricate spiraling trajectories around the spindle, much like a thread spun from a yarn. At the same time, they would swing
John Hipple in 1943. Image reproduced from Encyclopedia of Mass Spectrometry: Vol. 9: Historical Perspectives, Part A: The Development of Mass Spectrometry (Keith A. Nier Alfred L. Yergey \& P. Jane Gale), Newnes, 2015, p. 112, with permission from Elsevier.

back and forth along the axis of the spindle, trapped in an electrostatic harmonic potential. The charge-to-mass ratio of

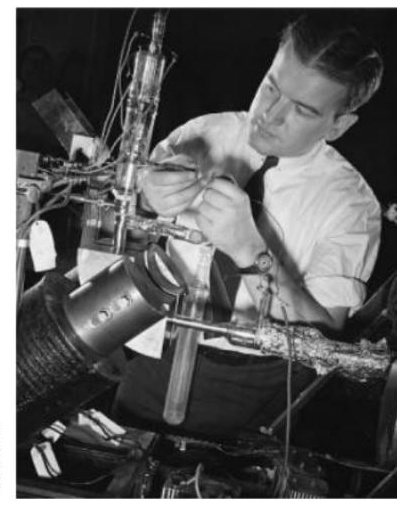
the ions can be derived from these harmonic axial oscillations. Combined with image current detection, as in FT-ICR, the orbitrap provides a high-accuracy, high-resolution, simple and compact mass analyzer that is now routinely used in proteomics research

(Milestone 20).

Whether achieved by using magnetic fields or electrostatic potentials, the idea of spinning ions on spiraling trajectories that betray their charge-to-mass ratio by the frequencies measured is a surprisingly simple yet very powerful concept. Iulia Georgescu, Senior Editor, Nature Physics

ORIGINAL RESEARCH PAPERS Hipple, J.A., Sommer, H. \& Thomas, H.A. A precise method of determining the Faraday by magnetic resonance. Phys. Rev. 76, 1877-1878 (1949)| Sommer, H., Thomas, H.A. \& Hipple, J.A. The measurement of eM by cyclotron resonance. Phys. Rev. 82, 697-702 (1951) | Comisarow, M.B. \& Marshall, A.G. Fourier transform ion cyclotron resonance spectroscopy. Chem. Phys. Lett. 25, 282283 (1974) | Makarov, A. Electrostatic axially harmonic orbital trapping: a high-performance technique of mass analysis. Anal. Chem. 72, 1156-1162 (2000)

FURTHER READING Kingdon, K.H. A method for the neutralization of electron space charge by positive ionization at very low gas pressures. Phys. Rev. 21, 408-418 (1923) 